\title{
Dietary habits mediate the relationship between socio-economic status and CVD factors among healthy adults: the ATTICA study
}

\author{
Demosthenes B Panagiotakos ${ }^{1, *}+$, Christos Pitsavos ${ }^{2}$, Christina Chrysohoou ${ }^{2}$, \\ Konstantinos Vlismas ${ }^{1}$, Yannis Skoumas ${ }^{2}$, Konstantina Palliou ${ }^{2}$ and Christodoulos Stefanadis ${ }^{2}$ \\ 'Department of Nutrition Science - Dietetics, Harokopio University, Athens, Greece: ${ }^{2}$ First Cardiology \\ Department, School of Medicine, University of Athens, Athens, Greece
}

Submitted 17 August 2007: Accepted 16 April 2008: First published online 11 July 2008

\begin{abstract}
Background: The aims of the present work were to investigate whether dietary habits are associated with socio-economic status (SES), and if they modify the relationship between SES and CVD risk factors, in a sample of men and women free from known CVD.

Methods: This population-based study was carried out in the province of Attica, where Athens is a major metropolis. During 2001-2002, information from 1528 men (18-87 years old) and 1514 women (18-89 years old) was collected (75\% participation rate). Among several sociodemographic, clinical and biological factors, adherence to the Mediterranean diet was assessed by a special diet score (Mediterranean Diet Score, MDS) that incorporated the inherent characteristics of this traditional diet. CVD risk factors were examined across the participants' educational level and annual income that defined their SES.

Results: Low SES groups exhibited higher prevalence of CVD risk factors, such as obesity, hypertension, diabetes mellitus and hypercholesterolaemia (all $P<0 \cdot 001)$. Low SES groups also showed less adherence to the Mediterranean diet than high SES groups (MDS: $23 \cdot 6$ (SD 8.1) v. $25 \cdot 6$ (SD 5.6), $P<0 \cdot 001$ ). Higher SES index was associated with lower likelihood of having hypercholesterolaemia $(\mathrm{OR}=0 \cdot 91 ; 95 \% \mathrm{CI} 0 \cdot 83,1 \cdot 00)$ and diabetes $(\mathrm{OR}=0 \cdot 83 ; 95 \% \mathrm{CI} 0 \cdot 72,0 \cdot 95)$, after adjusting for various potential confounders. However, the previously mentioned inverse relationship observed between SES and prevalence of CVD risk factors was mainly explained by the dietary habits of the participants.

Conclusions: Low SES groups showed less adherence to the Mediterranean diet compared with high SES groups. This finding may, in part, explain the higher CVD risk factors profile observed among low SES participants.
\end{abstract}

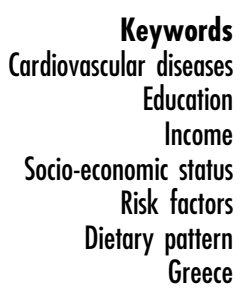

Much evidence indicates that the beneficial trends in CVD mortality have not been felt equally across all segments of society. Several observational studies have shown that the socio-economic status (SES) of adults, mainly determined from occupation, formal educational level and income, is related to mortality and morbidity from CVD and other chronic diseases, especially in industrialized countries $^{(1-5)}$. It has been suggested that the most striking improvements in CVD health have occurred among wealthier, better educated people, while progress among groups with lower SES has lagged ${ }^{(1)}$. Moreover, at the beginning of the 20th century results from observational studies suggested that CVD was originally more common in the upper socio-economic class than the lower or

† Correspondence address: 46 Paleon Polemiston Street, Glyfada 166 74, Attica, Greece. middle class (i.e. a 'disease of affluence') $)^{(1)}$. This was attributed mainly to the increased prevalence of the common CVD risk factors (e.g. smoking habits, sedentary life, obesity, etc.) in individuals with higher education and income. However, after the middle of the 20th century this changed gradually, especially in Westernized countries, so that CVD is currently more common in lower socio-economic groups ${ }^{(6-9)}$. Moreover, nowadays observational studies show that low SES individuals are associated with increased prevalence of CVD risk factors, such as hypertension, sedentary life, unhealthy dietary habits, cigarette smoking and high cholesterol ${ }^{(10-13)}$. It has been hypothesized that dietary choices may be one of the many factors mediating the relationship between social class and CVD risk. Changes in dietary patterns by social class may partly explain the shift in the social class distribution of CVD that seems to have occurred during 
the 1950 s and $1960 \mathrm{~s}^{(6)}$. It is known that a variety of social, economic, educational and cultural factors can influence the type of food consumed. Income and education have been found to be inversely related to atherogenic diets and trends over time in diet differed according to socioeconomic group ${ }^{(7)}$. The relationship between socio-economic factors and diet has been examined on the basis of food and nutrient intake and the results are mixed, sometimes contradictory, and often the observed differences are small ${ }^{(3)}$. Whenever differences are found, it is usually the case that persons from socio-economically disadvantaged backgrounds have food intake consistent with their higher rates of chronic disease ${ }^{(4,5)}$.

Greece was known in the mid-1960s and 1970s for its low incidence of CVD among Western populations, mainly due to the different lifestyle-related behaviours, like dietary habits ${ }^{(14)}$. However, during past decades the Greek population has experienced marked but uneven socio-economic development. Although education status has improved, the lifestyle of people has worsened and age-old dietary habits and high habitual physical activity have gradually given way to 'Western'-type diets and a more sedentary lifestyle, with increased smoking habits. An inverse relationship between education status and prevalence of CVD in the Greek population was recently reported by the CARDIO2000 investigators ${ }^{(15)}$. However, whether this association may be attributed to differences in dietary habits has never been reported before. Therefore, in the present work we sought to investigate whether dietary habits are associated with SES, and if they influence the relationship between SES and CVD risk factors, in a sample of Greek men and women free from known CVD.

\section{Methods}

\section{Subjects}

The ATTICA epidemiological study ${ }^{(16)}$ has been carried out in the province of Attica (including $78 \%$ urban and $22 \%$ rural areas), where Athens is a major metropolis. From May 2001 to December 2002, 4056 inhabitants from the above area were selected to enrol into the study. The sampling was random and stratified according to the age-gender-city distribution of the reference population (census 2001). Of the 4056 inhabitants, 3042 agreed to participate ( $75 \%$ participation rate). The sample is considered representative of the above-mentioned area (which covers about $50 \%$ of the total population), but not of the entire country. All participants were interviewed face-to-face, by trained personnel (cardiologists, general practitioners, dietitians and nurses) who used a standard questionnaire; men and women were excluded from the study if they reported a history of CVD or any other atherosclerotic disease, as well as chronic viral infections. Moreover, participants did not have cold or flu, acute respiratory infection, dental problems or any type of surgery in the past week. Also, all people living in institutions were excluded from the sampling.

Power analysis showed that the number of enrolled participants is adequate to evaluate two-sided standardized differences between subgroups of the study and the investigated parameters greater than 0.5 , achieving statistical power greater than 0.90 at $5 \%$ probability level ( $P$ value).

The study was approved by the Medical Research Ethics Committee of our institution and was carried out in accordance with the Declaration of Helsinki (1989) of the World Medical Association.

\section{Ascertainment of socio-economic status}

SES was assessed through educational level in three groups: (i) low education, $<9$ years of schooling (18.5\% of the total population); (ii) medium education, 9-12 years of schooling ( $45.8 \%$ of the total population); and (iii) high, $>12$ years of schooling $(35.7 \%$ of the total population); and mean annual income of the family (through self-reports) during the last three years, also in three groups: (i) low income, $<8000 €(20 \cdot 8 \%$ of the total population); (ii) medium income, 8000-10000 $€(33.3 \%$ of the total population); and (iii) high income, $>10000 €$ $(45.9 \%$ of the total population). To provide a more accurate estimate of peoples' SES we decided to combine income and education. Therefore, participants were classified into the following nine categories (classes): (i) low education and low income $(5.9 \%$ of the total population); (ii) low education and medium income $(5 \cdot 8 \%$ of the total population); (iii) low education and high income ( $3.6 \%$ of the total population); (iv) medium education and low income $(10.7 \%$ of the total population); (v) medium education and medium income $(18.1 \%$ of the total population); (vi) medium education and high income (19.0\% of the total population); (vii) high education and low income ( $4 \cdot 2 \%$ of the total population); (viii) high education and medium income $(9 \cdot 4 \%$ of the total population); and (ix) high education and high income $(23.4 \%$ of the total population).

At this point it should be discussed that, in order to evaluate the research hypothesis, a complex SES index was developed combining education (first) and income (after). Many scientists have suggested that stratification first by education and afterwards by income provides a better understanding of individuals' current SES. For example, a highly educated person might, at a particular phase of his/her life course, be unemployed, thus having a low income. Furthermore, stratification using only one indicator may yield misleading results or provide less information than using multiple measures ${ }^{(6)}$.

\section{Other variables investigated}

The evaluation of nutritional habits was based on a validated semi-quantitative FFQ (the EPIC-Greek FFQ, used in the European Prospective Investigation into Cancer and Nutrition, kindly provided by the Unit of Nutritional 
Epidemiology of Athens Medical School) ${ }^{(17)}$ that included all major food groups (i.e. cereals, fruits, vegetables, greens, legumes, potatoes, fish and fish products, meat and meat products, poultry, dairy products, sweets, etc.), as well as use of olive oil or other added fat (butter, margarine, etc.) and consumption of various types of beverages. In particular, the consumption of 156 food items and the portion size as an average per week was recorded. Then, the frequency of consumption was quantified approximately in terms of the number of times per month the food was consumed. In order to describe overall dietary habits we used the Mediterranean Diet Score (MDS) ${ }^{(18)}$. Briefly, the score includes ascertainment of ten major food groups and alcohol intake. Using discrete monotonic functions ranging from 0 to 5 , which are assigned to the frequency of consumption of foods that are recommended on a daily or weekly basis in the dietary guidelines based on the traditional Mediterranean diet pyramid ${ }^{(19)}$ (like fruits, vegetables, cereals, greens, legumes, fish, etc.), and inverse functions ranging from 5 to 0 for foods that are recommended on a monthly basis (i.e. meat and meat products, poultry, full-fat dairy products), a total score (range from 0 to 55) was developed. Higher values of the score indicate better adherence to this traditional dietary pattern ${ }^{(18)}$. For alcohol we assigned a score of 5 for consumption of less than 3 glasses/d (1 glass was considered equal to $100 \mathrm{ml}$ of ethanol, $12 \%$ concentration), a score of 0 for consumption of more than 7 glasses/d, and scores of 1 to 4 for consumption of 3 , 4-5, 6 and 7 glasses/d respectively.

We used a translated version of the International Physical Activity Questionnaire (IPAQ), suitable for assessing population levels of self-reported physical activity ${ }^{(20)}$. The short version of IPAQ (nine items) that we used provided information on weekly time spent walking, in vigorousintensity, moderate-intensity and sedentary activity. Participants were instructed to refer to all domains of physical activity during a usual week of the past year. Participants were classified as inactive when no physical activity was reported. IPAQ has been used previously in the Greek population and has been found valid to assess energy expenditure (unpublished data).

Current smokers were defined as those who smoked at least one cigarette on a daily basis or had stopped cigarette smoking during the past 12 months. Former smokers were defined as those who had stopped smoking more than 1 year previously. The remainder were defined as never smokers. Standing height and weight were measured and BMI was calculated as weight divided by the square of height $\left(\mathrm{kg} / \mathrm{m}^{2}\right)$. Obesity was defined according to the WHO criteria as BMI $>29 \cdot 9 \mathrm{~kg} / \mathrm{m}^{2}$. Resting blood pressure was measured after the subject had been sitting comfortably for 5-10 min and with the cuff arm supported at heart level. Hypertension was defined as systolic blood pressure $\geq 140 \mathrm{mmHg}$, or diastolic blood pressure $\geq 90 \mathrm{mmHg}$, or the use of any antihypertensive medication.
Hypercholesterolaemia was defined as serum total cholesterol level $>200 \mathrm{mg} / \mathrm{dl}$ or the use of lipid-lowering medication; and diabetes mellitus as fasting blood glucose $>125 \mathrm{mg} / \mathrm{dl}$ or the use of antidiabetic medication. Finally, we asked all participants: 'Have you ever been told to use a medication due to high blood pressure, or lipids or blood glucose levels?' If 'yes', then we asked them whether they followed the recommendations of their physician. Participants who answered 'no' in the last question were classified as non-compliers.

Further details regarding the sampling, the procedures and the measurements of the ATTICA study may be found elsewhere $^{(16)}$.

\section{Statistical analyses}

Continuous variables are presented as means and standard deviations, while categorical variables are presented as frequencies. Comparisons between normally distributed continuous variables across groups of participants were performed by ANOVA, after controlling for equality of variances. However, multiple comparisons were made and thus we used the Bonferroni rule in order to account for the increase in type I error. Nested multiple logistic regression models were estimated having prevalence of CVD risk factors as dependent variables, SES indices as the main effect and various characteristics of the participants (together with dietary habits as assessed through the MDS) as covariates. Deviance residuals and the HosmerLemeshow statistic were used to evaluated models' goodness-of-fit. All reported $P$ values are based on two-sided tests and compared with a significance level of $5 \%$. The Statistical Package for the Social Sciences statistical software package version 13 (SPSS Inc., Chicago, IL, USA) was used for all statistical calculations.

\section{Results}

Table 1 illustrates various characteristics of the participants by education and income level. Regarding age distribution there was a significant difference $(P<0 \cdot 0001)$ between groups, with the low education groups being significantly older than the medium and high education groups. Additionally, gender distribution showed that the low income groups in all education classes comprised mainly women $(P<0 \cdot 0001)$. Regarding CVD risk factors, comparisons between SES groups showed that the low and medium education groups had a higher prevalence of CVD risk factors than the high education groups. Moreover, physical activity assessment showed that the most physically active groups were people with high education. Smoking was prevalent in all groups, with the low education/low income group exhibiting the lowest percentage of all groups and the medium education/medium income group exhibiting the highest. Moreover, hypertension, diabetes mellitus and hypercholesterolaemia 


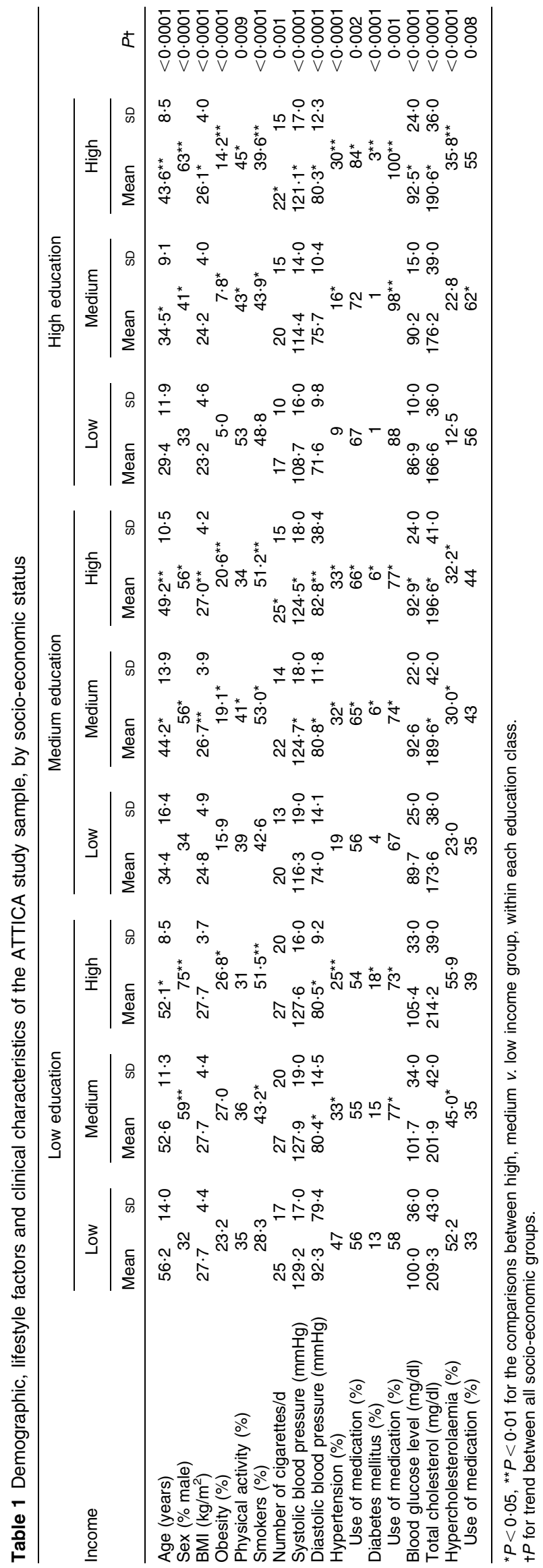

were more prevalent in the low education groups, across all income classes. Figure 1 illustrates the average level of CVD risk factors (hypertension, diabetes, hypercholesterolaemia and obesity, range 0-4) in the study's participants.

The weekly consumption of food groups and beverages is illustrated in Table 2. Compared with the dietary guidelines based on the traditional Mediterranean diet pyramid $^{(17)}$, it was observed that the consumption of red meat was much higher than recommended (i.e. 1 serving/ week) in all groups, whereas the consumption of poultry was much less than the recommended intake. In addition, fish consumption was lower than the recommended (i.e. 5-6 servings/week) in all SES groups. Regarding dairy products and cereals the consumption was close to the dietary guidelines (i.e. 1-2 servings/d and 8 servings/d, respectively). Consumption of legumes, fruits, potatoes and sweets was higher than recommended (recommendations: 3-4 servings/week for legumes, 3 servings/d for fruits, 3 servings/week for potatoes and 3 servings/week for sweets). On the other hand, consumption of vegetables was much lower than the recommended intake (i.e. 6 servings/d). Alcohol drinking was close to the recommended intake, but differed significantly between all SES levels with increased intake observed in the low and medium education groups. No significant differences were observed between SES groups regarding energy intake. Finally, higher adherence to the Mediterranean diet was observed in the medium and high SES groups compared with the low SES group.

To further evaluate the association between indices of SES (income, education) and prevalence of diabetes, hypertension and hypercholesterolaemia, we applied nested multiple logistic regression models (Table 3). In model 1 higher income was inversely associated with the prevalence of CVD risk factors (i.e. hypertension, hypercholesterolaemia and diabetes). Similarly, in models 2 and 3, higher education status as well as higher SES index was inversely associated with the prevalence of the aforementioned CVD risk factors, even after adjusting for age, gender, smoking habits, BMI, physical activity status and compliance to medication. However, SES index lost its significance when dietary habits were taken into account. In particular, the effect size of SES (i.e. odds ratios) on the previously mentioned outcomes was no longer significant when MDS and energy intake were also included in the models (model 4). Moreover, the interaction terms between SES and MDS were highly significant regarding systolic blood pressure $(P<0 \cdot 001)$, blood glucose $(P<0 \cdot 001)$ and serum total cholesterol levels $(P<0 \cdot 001)$.

\section{Discussion}

In the present work we studied the relationship between SES and CVD risk factors in relation to dietary habits in a Greek sample of men and women free from known 


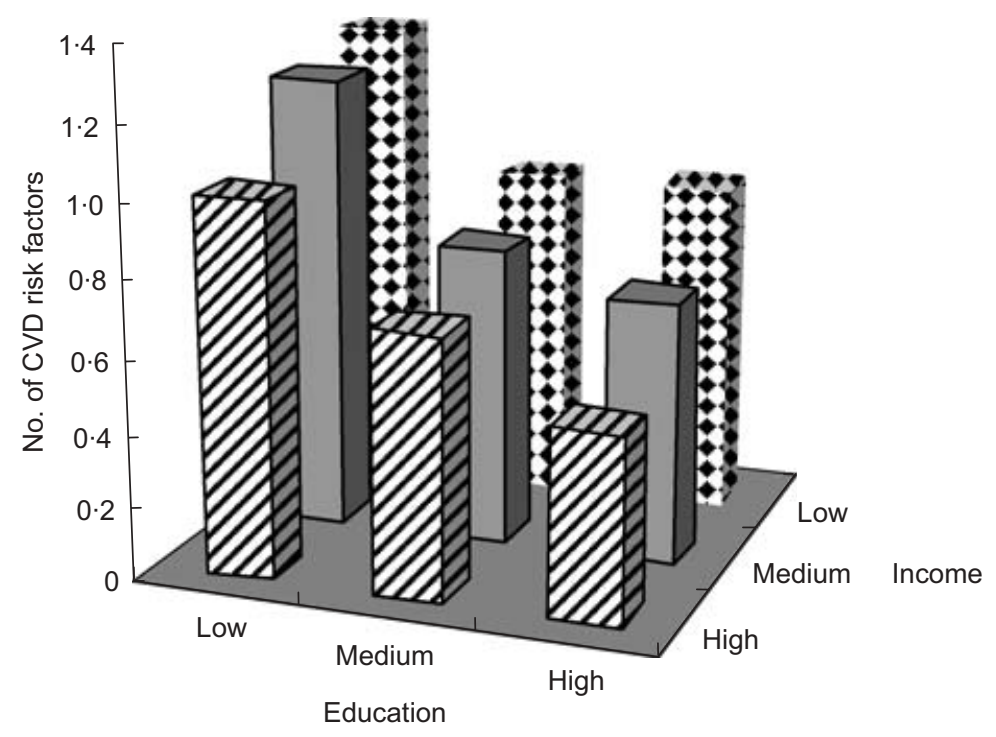

Fig. 1 Average number of CVD risk factors (hypertension, obesity, diabetes and hypercholesterolaemia) by education and income among the ATTICA study participants

CVD or other chronic diseases. The results revealed that although participants are trying to comply with the dietary guidelines (adequate intakes of fruits, legumes, cereals and low-fat dairy products), overall they do not follow the traditional Mediterranean diet, especially people belonging to the low SES groups. The latter finding explains, in part, the observed inverse association between the prevalence of CVD risk factors and SES of the participants.

In order to define SES three indicators have most often been used: occupation, education and income. However, the relatively weak correlation between these three indices in developed countries (correlation coefficients range from 0.3 to 0.6 ) suggests that although these tools measure the same concept, they cover different aspects of the socio-economic structure contributing individually to the relationship between SES and disease ${ }^{(21)}$. Moreover, it has not yet been defined which social factor is most suitable to determine the social level and its relationship to health status. Taking into account that in the ATTICA study there was not sufficient information concerning the occupational status of the participants, we decided to combine two of the three features of SES (i.e. education and income) in evaluating our research hypothesis.

Analysis of lifestyle characteristics of the participants showed that a great many of them were defined as smokers, with the high education group smoking less than the rest. Furthermore, a large part of the sample followed a sedentary lifestyle, with the low SES participants being more inactive than the others. A consistent relationship was observed between prevalence of CVD risk factors and SES group. In particular, individuals belonging in the lower SES classes had increased levels of blood pressure, lipids, glucose and BMI (Table 1). The inverse relationship between SES and CVD risk factors levels was found consistently in multi-adjusted analyses that controlled for age, gender, smoking habits, BMI, physical activity status and compliance to medication, but not for dietary habits.

Regarding dietary characteristics, the MDS was much lower in people assigned in the low classes of the SES spectrum, as compared with the higher classes. Particularly, people in the low SES groups consumed more meat and products, more dairy products (full in fat), they preferred to use margarine and butter in daily cooking and meals instead of olive oil, and they drank alcoholic beverages and coffee more frequently. Several studies have already underlined that the consumption of a healthy dietary pattern that includes cereals, fruits and vegetables and low-fat dairy products is more common in high SES groups, while intake of foods rich in fat and energy is more common in those of low SES ${ }^{(22)}$. However, at this point it should be mentioned that the consumption of energy-rich food is considered beneficial by low rank people, when manual work is involved. Regarding alcohol drinking, several studies have reported an inverse relationship with $\operatorname{SES}^{(7,23-25)}$. Alcohol intake was satisfactory in our study, but it was significantly different between SES groups, with the high education/low income group having the lowest consumption and the low education/high income group having the highest intake (Table 2). This could be explained by the gender distribution difference, as the first group had a significantly lower percentage of males present compared with the other group.

As mentioned above, the prevalence of common CVD risk factors, such as obesity, hypertension, diabetes mellitus and hypercholesterolaemia, was higher in the low SES groups compared with the high SES groups. A major research hypothesis in this work was to test whether 
Table 2 Food group consumption (servings/week unless stated otherwise) of the ATTICA study sample, by socio-economic status

\begin{tabular}{|c|c|c|c|c|c|c|c|c|c|c|c|c|c|c|c|c|c|c|c|}
\hline \multirow{3}{*}{ Income } & \multicolumn{6}{|c|}{ Low education } & \multicolumn{6}{|c|}{ Medium education } & \multicolumn{6}{|c|}{ High education } & \multirow[b]{3}{*}{$P \dagger$} \\
\hline & \multicolumn{2}{|c|}{ Low } & \multicolumn{2}{|c|}{ Medium } & \multicolumn{2}{|c|}{ High } & \multicolumn{2}{|c|}{ Low } & \multicolumn{2}{|c|}{ Medium } & \multicolumn{2}{|c|}{ High } & \multicolumn{2}{|c|}{ Low } & \multicolumn{2}{|c|}{ Medium } & \multicolumn{2}{|c|}{ High } & \\
\hline & Mean & SD & Mean & SD & Mean & SD & Mean & SD & Mean & SD & Mean & SD & Mean & SD & Mean & SD & Mean & SD & \\
\hline Red meat and products & $5 \cdot 1 \ddagger$ & $2 \cdot 8$ & $5 \cdot 3 \ddagger$ & $3 \cdot 4$ & $5 \cdot 0 \ddagger$ & $2 \cdot 0$ & $5 \cdot 6 \ddagger$ & $3 \cdot 5$ & $4 \cdot 9 \ddagger^{*}$ & $2 \cdot 4$ & $4 \cdot 5 \ddagger^{\star \star}$ & $2 \cdot 3$ & $4 \cdot 7 \ddagger$ & $2 \cdot 1$ & $4 \cdot 2 \ddagger^{\star}$ & $2 \cdot 2$ & $4 \cdot 4 \ddagger$ & 1.9 & 0.001 \\
\hline Poultry & $1 \cdot 8 \neq$ & $0 \cdot 6$ & $1 \cdot 4 \ddagger$ & $1 \cdot 1$ & $1 \cdot 1 \ddagger$ & 0.4 & $1 \cdot 5 \ddagger$ & 0.9 & $1 \cdot 3 \ddagger$ & $0 \cdot 8$ & $1 \cdot 2 \ddagger^{*}$ & $0 \cdot 6$ & $1 \cdot 6 \ddagger$ & $1 \cdot 1$ & $1 \cdot 4 \ddagger$ & $0 \cdot 8$ & $1 \cdot 3 \ddagger^{*}$ & $0 \cdot 6$ & 0.004 \\
\hline Fish & $2 \cdot 3 \ddagger$ & $2 \cdot 0$ & $2 \cdot 0 \ddagger$ & $1 \cdot 0$ & $1 \cdot 7 \ddagger$ & $0 \cdot 9$ & $2 \cdot 0 \ddagger$ & $1 \cdot 3$ & $2 \cdot 0 \ddagger$ & $1 \cdot 4$ & $2 \cdot 2 \ddagger$ & $1 \cdot 3$ & $2 \cdot 1 \ddagger$ & $1 \cdot 2$ & $2 \cdot 2 \ddagger$ & $1 \cdot 4$ & $2 \cdot 3 \ddagger$ & $1 \cdot 2$ & $0 \cdot 30$ \\
\hline Legumes & $5 \cdot 7$ & $4 \cdot 6$ & $5 \cdot 3$ & $2 \cdot 9$ & $5 \cdot 2$ & $1 \cdot 8$ & $5 \cdot 1$ & $2 \cdot 9$ & $5 \cdot 0$ & $2 \cdot 7$ & $5 \cdot 0$ & $2 \cdot 3$ & $4 \cdot 6$ & $2 \cdot 8$ & $5 \cdot 2$ & $4 \cdot 1$ & $5 \cdot 1$ & $2 \cdot 2$ & $0 \cdot 90$ \\
\hline Dairy products & $12 \cdot 4$ & $6 \cdot 2$ & $11 \cdot 6$ & $6 \cdot 9$ & $11 \cdot 0$ & $4 \cdot 1$ & $13 \cdot 3$ & $6 \cdot 4$ & $11 \cdot 1^{\star}$ & $5 \cdot 3$ & $10 \cdot 9^{*}$ & $4 \cdot 4$ & $12 \cdot 8$ & $4 \cdot 6$ & $12 \cdot 0$ & $4 \cdot 7$ & $11 \cdot 5$ & $4 \cdot 2$ & 0.008 \\
\hline Fruits & $28 \cdot 4$ & $16 \cdot 2$ & $29 \cdot 7$ & $16 \cdot 6$ & $27 \cdot 6$ & $13 \cdot 2$ & $27 \cdot 2$ & $16 \cdot 8$ & $24 \cdot 9$ & $13 \cdot 0$ & $26 \cdot 3$ & $12 \cdot 1$ & $26 \cdot 9$ & $14 \cdot 6$ & $26 \cdot 1$ & $13 \cdot 8$ & $25 \cdot 7$ & $11 \cdot 9$ & 0.69 \\
\hline Vegetables & $35 \cdot 4$ & $23 \cdot 2$ & $37 \cdot 0$ & $15 \cdot 4$ & $31 \cdot 6 \neq$ & $10 \cdot 1$ & $30 \cdot 0 \ddagger$ & $16 \cdot 0$ & $31 \cdot 7 \ddagger$ & $13 \cdot 3$ & 33.9 & $13 \cdot 7$ & $32 \cdot 6 \ddagger$ & $12 \cdot 5$ & $33 \cdot 5$ & $14 \cdot 5$ & $35 \cdot 6$ & $13 \cdot 9$ & $0 \cdot 39$ \\
\hline Potatoes & $11 \cdot 9$ & $8 \cdot 7$ & $13 \cdot 4$ & $9 \cdot 2$ & $12 \cdot 0$ & $6 \cdot 1$ & $14 \cdot 4$ & $9 \cdot 1$ & $11 \cdot 9^{* *}$ & $6 \cdot 5$ & $11 \cdot 4^{\star *}$ & $6 \cdot 5$ & $12 \cdot 2$ & $6 \cdot 0$ & $11 \cdot 3^{*}$ & $7 \cdot 0$ & $10 \cdot 5^{\star}$ & $5 \cdot 4$ & 0.001 \\
\hline Cereals & $62 \cdot 4$ & $28 \cdot 6$ & $58 \cdot 3$ & $27 \cdot 3$ & $42 \cdot 7 \ddagger^{\star \star}$ & * $15 \cdot 3$ & $51 \cdot 8$ & $18 \cdot 0$ & $51 \cdot 0$ & $16 \cdot 8$ & $52 \cdot 6$ & $18 \cdot 0$ & $50 \cdot 1$ & $19 \cdot 3$ & $50 \cdot 9$ & $17 \cdot 6$ & $52 \cdot 5$ & $14 \cdot 2$ & 0.008 \\
\hline Sweets & $5 \cdot 1$ & $3 \cdot 5$ & $5 \cdot 1$ & $3 \cdot 1$ & $4 \cdot 4$ & 1.9 & $5 \cdot 3$ & $2 \cdot 4$ & 4.5 & $2 \cdot 3$ & 4.9 & $2 \cdot 3$ & $5 \cdot 1$ & $2 \cdot 0$ & $5 \cdot 0$ & $2 \cdot 4$ & $5 \cdot 0$ & $2 \cdot 1$ & 0.295 \\
\hline Coffee $(\mathrm{ml} / \mathrm{d})$ & $88 \cdot 2$ & $99 \cdot 1$ & $140 \cdot 2$ & $157 \cdot 1$ & $167 \cdot 7^{\star \star}$ & $153 \cdot 2$ & $151 \cdot 8$ & $108 \cdot 1$ & $133 \cdot 2$ & $140 \cdot 2$ & $121 \cdot 9^{\star}$ & $124 \cdot 9$ & $115 \cdot 8$ & $102 \cdot 0$ & $132 \cdot 7^{\star}$ & $121 \cdot 0$ & \multirow{2}{*}{\multicolumn{2}{|c|}{$\begin{array}{c}130 \cdot 2^{* *} 115 \cdot 2 \\
21^{*}\end{array}$}} & 0.001 \\
\hline Alcohol drinking (\%) & \multicolumn{2}{|c|}{18} & \multicolumn{2}{|c|}{$44^{\star \star}$} & $30^{*}$ & & \multicolumn{2}{|c|}{20} & \multicolumn{2}{|c|}{$36^{*}$} & \multicolumn{2}{|c|}{$32^{*}$} & \multicolumn{2}{|c|}{27} & \multicolumn{2}{|c|}{$20^{*}$} & & & 0.002 \\
\hline Ethanol intake $(g / d)$ & $8 \cdot 0$ & $11 \cdot 2$ & $9 \cdot 3^{* *}$ & $12 \cdot 1$ & $14 \cdot 6^{\star \star}$ & $26 \cdot 8$ & \multirow{2}{*}{\multicolumn{2}{|c|}{$\begin{array}{c}4 \cdot 8 \\
10 \cdot 15\end{array}$}} & $11 \cdot 2^{* *}$ & $18 \cdot 7$ & $8 \cdot 7^{\star \star}$ & $12 \cdot 0$ & $4 \cdot 2$ & $5 \cdot 8$ & \multirow{2}{*}{\multicolumn{2}{|c|}{$\begin{array}{l}5 \cdot 3 \\
9 \cdot 37\end{array}$}} & \multicolumn{2}{|c|}{$5 \cdot 8^{\star \star} \quad 4 \cdot 1$} & $<0.0001$ \\
\hline Energy intake (MJ/d) & $9 \cdot 64$ & $4 \cdot 01$ & $10 \cdot 91$ & $4 \cdot 68$ & $10 \cdot 22$ & 3.94 & & & $9 \cdot 91$ & $4 \cdot 11$ & $9 \cdot 36$ & $3 \cdot 91$ & $9 \cdot 73$ & $3 \cdot 64$ & & & 9.54 & $3 \cdot 49$ & 0.39 \\
\hline Energy intake $(\mathrm{kcal} / \mathrm{d})$ & 2302 & 958 & 2606 & 1117 & 2442 & 941 & \multirow{2}{*}{\multicolumn{2}{|c|}{$\begin{array}{c}2425{ }^{1} 1008 \\
67\end{array}$}} & \multirow{2}{*}{\multicolumn{2}{|c|}{$23687^{982}$}} & 2236 & 935 & \multirow{2}{*}{\multicolumn{2}{|c|}{$\begin{array}{c}2325{ }^{870} \\
89\end{array}$}} & 2239 & 925 & 2278 & 834 & 0.39 \\
\hline Use of olive oil (\%) & 57 & & $7 C$ & $b^{*}$ & 58 & & & & & & 79 & & & & 90 & 0 & 95 & & 0.001 \\
\hline Use of margarine (\%) & 65 & & 5 & 7 & $89^{*}$ & & & 68 & 79 & & 45 & & 5 & 57 & 45 & 15 & 35 & & 0.001 \\
\hline MDS (0-55) & $23 \cdot 6$ & $8 \cdot 1$ & $24 \cdot 2$ & $6 \cdot 1$ & $23 \cdot 9$ & $6 \cdot 6$ & $29 \cdot 4$ & $8 \cdot 7$ & $25 \cdot 7^{*}$ & $7 \cdot 2$ & $24 \cdot 9^{\star *}$ & $6 \cdot 8$ & $29 \cdot 9$ & $8 \cdot 7$ & $27 \cdot 6^{\star}$ & $5 \cdot 9$ & $25 \cdot 6^{*}$ & $5 \cdot 6$ & $<0.0001$ \\
\hline
\end{tabular}

MDS, Mediterranean Diet Score (incorporates the inherent characteristics of the Mediterranean type of diet; higher values of the score indicates greater adherence to this traditional dietary pattern).

${ }^{*} P<0.05,{ }^{*} P<0.01$ for the comparisons between high, medium $v$. low income group, within each education class.

$+P$ for trend between all socio-economic groups.

$\ddagger P<0.05$ for the comparisons between the reported consumption of various food groups and alcohol, with the recommendations for the Greek population ${ }^{(18)}$. 
Table 3 Results from multiple logistic regression analysis regarding the association between indices of socio-economic status (SES; income and education) on the prevalence of hypertension, hypercholesterolaemia and diabetes, adjusted for lifestyle variables, of the ATTICA study sample

\begin{tabular}{|c|c|c|c|c|c|c|c|c|}
\hline & \multicolumn{2}{|c|}{ Model 1} & \multicolumn{2}{|c|}{ Model 2} & \multicolumn{2}{|c|}{ Model 3} & \multicolumn{2}{|c|}{ Model 4} \\
\hline & OR & $95 \% \mathrm{Cl}$ & OR & $95 \% \mathrm{Cl}$ & OR & $95 \% \mathrm{Cl}$ & OR & $95 \% \mathrm{Cl}$ \\
\hline \multicolumn{9}{|c|}{ Dependent variable: Hypertension } \\
\hline Income (high $v$. low) & 0.98 & $0.94,1.00$ & - & & - & & - & \\
\hline Education (high $v$. low) & - & & $1 \cdot 01$ & $0.58,1.77$ & - & & - & \\
\hline SES index $(1-9)$ & - & & - & & $1 \cdot 06$ & $0 \cdot 95,1 \cdot 18$ & 1.09 & $0 \cdot 89,1 \cdot 33$ \\
\hline \multicolumn{9}{|c|}{ Dependent variable: Hypercholesterolaemia } \\
\hline Income (high $v$. low) & 0.58 & $0.39,0.85$ & - & & - & & - & \\
\hline Education (high v. low) & - & & $0 \cdot 70$ & $0.52,0.93$ & - & & - & \\
\hline SES index $(1-9)$ & - & & - & & 0.91 & $0 \cdot 83,1 \cdot 00$ & 0.92 & $0 \cdot 78,1 \cdot 07$ \\
\hline \multicolumn{9}{|c|}{ Dependent variable: Diabetes } \\
\hline Income (high $v$. low) & $0 \cdot 14$ & $0.05,0.37$ & - & & - & & - & \\
\hline Education (high $v$. low) & - & & 0.44 & $0 \cdot 21,0.88$ & - & & - & \\
\hline SES index $(1-9)$ & - & & - & & 0.83 & $0.72,0.95$ & 0.89 & $0 \cdot 68,1 \cdot 15$ \\
\hline
\end{tabular}

Model 1 includes income, as well as age, gender, smoking habits, BMI, physical activity status and compliance to medication. Model 2 includes education, as well as the aforementioned covariates. Model 3 includes SES (as an ordinal variable 1-9), as well as the aforementioned covariates. Model 4 includes SES index, Mediterranean Diet Score, energy intake, as well as the aforementioned covariates.

dietary habits play a role in the aforementioned inverse relationship. Nested linear regression models revealed that when the diet component was included in the analysis (i.e. the MDS), the relationship between SES and CVD risk factors levels did not reach significance (Table 3). It is known that education may facilitate the acquisition of positive social, psychological and economic skills and assets, and may provide insulation from adverse influences, thus leading to positive attitudes about health ${ }^{(26,27)}$. Better educated people, with higher income, may adhere to a healthier dietary pattern. The latter suggests that there might be a need for a nutritional education programme among low and medium SES groups, in order to lower the burden of CVD.

\section{Limitations}

The present study was cross-sectional in nature and shares all the limitations of this study type (i.e. recall bias, lack of causal inference). Another potential limitation, common to most nationwide population social surveys, is that the poor were not well represented, because homeless, unemployed or migrants not speaking the dominant language are difficult to reach and often specially tailored methods are needed to obtain accurate dietary data from these groups. Also, as in all studies that use self-reported data, social desirability reporting bias was possible, especially in the higher SES groups, since the better educated class is more likely to be conscious of desirable eating habits. Finally, there is no exact measurement of SES in Greece. This may limit our findings since people from various SES groups may have been misclassified.

\section{Conclusion}

We have studied the role of dietary habits in the relationship between SES and CVD risk factors among apparently healthy men and women living in a Mediterranean country. Results showed that overall the participants do not follow the traditional Mediterranean diet, especially those classified as low SES. Additionally, a novel finding of the present work is that dietary habits seem to modify the inverse relationship observed between SES and CVD risk factor levels. Thus, much attention should be given to people of low SES, in order to reduce the burden of future CVD.

\section{Acknowledgements}

The ATTICA study is funded by research grants from the Hellenic Society of Cardiology. The authors would like to thank the investigators of the ATTICA study: A. Zeimbekis (physical examination), N. Papaioannou (physical examination), E. Tsetsekou (physical evaluation), L. Papadimitriou (physical evaluation), N. Massoura (physical examination), S. Vellas (physical examination), A. Katinioti (physical examination), M. Toutouza (data management), M. Kambaxis (dietary assessment), C. Tselika (technical support) and S. Poulopoulou (technical support).

Conflicts of interest: None.

Contribution of each author: D.B.P. designed the study, performed the data analysis and wrote the paper; C.P. designed the study and reviewed the paper; C.C. designed the study and performed the clinical evaluation; K.V. reviewed the paper; Y.S. performed the clinical evaluation; K.P. performed the dietary evaluation; C.S. critically reviewed the paper and supervised the study.

\section{References}

1. Lantz PM, Lynch JW, House JS, Lepkowski JM, Mero RP, Musick MA \& Williams DR (2001) Socioeconomic disparities in health change in a longitudinal study of US adults: the role of health-risk behaviours. Soc Sci Med 53, 29-40. 
2. Gran B (1995) Major differences in cardiovascular risk indicators by educational status. Results from a population based screening program. Scand J Soc Med 23, 9-16.

3. Bucher HC \& Ragland DR (1995) Socioeconomic indicators and mortality from coronary heart disease and cancer: a 22-year follow-up of middle-aged men. Am J Public Health 85, 1231-1236.

4. Cooper R (2001) Social inequality, ethnicity and cardiovascular disease. Int J Epidemiol 30, 48-52.

5. Woodward M, Shewry MC, Smith WCS \& Tunstall-Pedoe H (1992) Social status and coronary heart disease: results from the Scottish Heart Health Study. Prev Med 21, 136-148.

6. McClelland JW, Keenan DP, Lewis J et al. (2001) Review of evaluation tools used to assess the impact of nutrition education on dietary intake and quality, weight management practices, and physical activity of low-income audiences. J Nutr Educ 33, Suppl. 1, S35-S48.

7. Irala-Estevez J, De Groth $\mathrm{M}$, Jahansson L, Oltersdof U, Prattala R \& Martinez-Gonzalez MA (2000) A systematic review of socio-economic differences in food habits in Europe: consumption of fruit and vegetables. Eur J Clin Nutr 54, 706-714.

8. Marmot MG, Adelstein AM \& Robinson N (1978) Changing social class distribution of heart disease. BMJ 2, 1109-1112.

9. Diez-Roux AV, Nieto JF, Caulfield L, Tyroler HA, Watson RL \& Szklo M (1999) Neighbourhood differences in diet: the Atherosclerosis Risk in Communities (ARIC). J Epidemiol Community Health 53, 55-63.

10. Luepker RV, Rosamond WD, Murphy R, Sprafka JM, Folsom AR, McGovern PG \& Blackburn H (1993) Socio-economic status and coronary heart disease risk factor trends. The Minnesota Heart Survey. Circulation 88, 2172-2179.

11. Sanchez-Villegas A, Martínez JA, Prättälä R, Toledo E, Roos G \& Martínez-González MA; FAIR-97-3096 Group (2003) A systematic review of socioeconomic differences in food habits in Europe: consumption of cheese and milk. Eur J Clin Nutr 57, 917-929.

12. López-Azpiazu I, Sánchez-Villegas A, Johansson L, Petkeviciene J, Prättälä R \& Martínez-González MA; FAIR-97-3096 Project (2003) Disparities in food habits in Europe: systematic review of educational and occupational differences in the intake of fat. J Hum Nutr Diet 16, 349-364.

13. Karasek RA, Theorell T, Schwartz JE, Schnall PL, Pieper CF \& Michela JL (1988) Job characteristics in relation to the prevalence of myocardial infarction in the US Health Examination Survey (HES) and Health Nutrition Examination Survey (HANES). Am J Public Health 78, 910-918.

14. Kalapothaki V, Kalantidi A, Katsouyanni K, Trichopoulou A, Kyriopoulos J, Kremastinou J, Hadjiconstantinou V \& Trichopoulos D (1992) The health of the Greek population. Materia Med Greca 20, 91-164.
15. Pitsavos C, Panagiotakos DB, Chrysohoou C, Skoumas J, Stefanadis C \& Toutouzas PK (2002) Education and acute coronary syndromes: results from the CARDIO2000 epidemiological study. Bull World Health Organ 80, 371-377.

16. Pitsavos C, Panagiotakos DB, Chrysohoou C \& Stefanadis C (2003) Epidemiology of cardiovascular risk factors, in Greece; aims, design and baseline characteristics of the ATTICA study. BMC Public Health 32, 9.

17. Katsouyanni K, Rimm EB, Gnardellis C, Trichopoulos D, Polychronopoulos E \& Trichopoulou A (1997) Reproducibility and relative validity of an extensive semi-quantitative food frequency questionnaire using dietary records and biochemical markers among Greek schoolteachers. Int J Epidemiol 26, Suppl. 1, S118-S127.

18. Panagiotakos DB, Pitsavos C \& Stefanadis C (2006) Dietary patterns: a Mediterranean diet score and its relation to clinical and biological markers of cardiovascular disease risk. Nutr Metab Cardiovasc Dis 16, 559-568.

19. Ministry of Health and Welfare, Supreme Scientific Health Council (1999) Dietary guidelines for adults in Greece. Arch Hellenic Med 16, 516-524.

20. Craig CL, Marshall AL, Sjostrom M et al. (2003) International physical activity questionnaire: 12-country reliability and validity. Med Sci Sports Exerc 35, 1381-1395.

21. Galobardes B, Morabia A \& Bernstein M (2001) Diet and socioeconomic position: does the use of different indicators matter? Int J Epidemiol 30, 334-340.

22. Giskes K, Turrell G, Patterson C \& Newman B (2002) Socioeconomic differences among Australian adults in consumption of fruit and vegetables and intakes of antioxidants and folate. J Hum Nutr Diet 15, 375-385.

23. Darmon N \& Khlat M (2002) An overview of the health status of migrants in France, in relation to their dietary practices. Public Health Nutr 4, 163-172.

24. Mortensen EL, Jensen HH, Sanders SA \& Reinisch JM (2006) Associations between volume of alcohol consumption and social status, intelligence, and personality in a sample of young adult Danes. Scand J Psychol 47, 387-398.

25. Romelsjö A (1989) The relationship between alcohol consumption and social status in Stockholm. Has the social pattern of alcohol consumption changed? Int J Epidemiol 18, 842-851.

26. Ball K, Crawford D \& Mishra G (2006) Socio-economic inequalities in women's fruit and vegetable intakes: a multilevel study of individual, social and environmental mediators. Public Health Nutr 9, 623-630.

27. Hulshof KF, Brussaard JH, Kruizinga AG, Telman J \& Lowik MRH (2003) Socioeconomic status, dietary intake and 10 y trends: the Dutch National Food Consumption Survey. Eur J Clin Nutr 57, 128-137. 